\title{
Sum frequency generation and catalytic reaction studies of the removal of the organic capping agents from Pt nanoparticles by UV-ozone treatment
}

\author{
Cesar Aliaga, ${ }^{\dagger}$ Jeong Y. Park, ${ }^{\dagger}$ Yusuke Yamada, $^{\dagger}{ }^{\#}$ Hyun Sook Lee, ${ }^{\dagger}$ Chia-Kuang \\ Tsung, ${ }^{\dagger}$ Peidong Yang, ${ }^{\dagger}$ and Gabor Somorjai. ${ }^{\dagger *}$ \\ † Department of Chemistry, University of California, Berkeley, CA 94720 and Materials \\ Sciences Division and Chemical Sciences Division, Lawrence Berkeley National \\ Laboratory, Berkeley, CA 94720
}

\begin{abstract}
We report the structure of the organic capping layers of platinum colloid nanoparticles and their removal by UV-ozone exposure. Sum frequency generation vibrational spectroscopy (SFGVS) studies identify the carbon-hydrogen stretching modes on poly(vinylpyrrolidone) (PVP), and tetradecyl tributylammonium bromide (TTAB) capped platinum nanoparticles. We found that the UV-ozone treatment technique effectively removes the capping layer, based on several analytical measurements including SFGVS, X-ray photoelectron spectroscopy, and Diffuse Reflectance Infrared Fourier Transform Spectroscopy (DRIFTS). The overall shape of the nanoparticles was preserved after the removal of capping layers, as confirmed by transmission electron microscopy (TEM). SFGVS of ethylene hydrogenation on the clean platinum nanoparticles demonstrates the existence of ethylidyne and di- $\sigma$-bonded species, indicating the similarity between single crystal and nanoparticle systems.
\end{abstract}

TITLE RUNNING HEAD: Sum Frequency Generation and Catalytic Reaction Studies of the removal of the organic capping agents from Pt nanoparticles by UV ozone treatment.

* Author to whom correspondence should be addressed. E-mail:somorjai@berkeley.edu

\# Current Address: Research Institute of Ubiquitous Energy Devices, National Institute of Advanced Industrial Science and Technology (AIST), 1-8-31 Midorigaoka, Ikeda, Osaka 563-8577 Japan. 


\section{Introduction.}

Industrial heterogeneous catalysts are usually composed of nanometer sized metallic clusters capped with diverse organic compounds, and supported by porous materials. Traditionally, fundamental catalysis studies are conducted on metal single crystals, which provide extensive information of the molecular processes at the surface, under optimal and well-controlled conditions. However, most of the industrial catalysts are highly dispersed nanoparticles and catalytic processes occur at elevated temperatures and high pressures. ${ }^{1,2}$ Therefore, it is essential to perform in-situ studies of model systems that have complexities associated with real catalytic conditions. ${ }^{3}$ The evolution of the science of nanomaterials has permitted the use of nanoparticles as model systems, ${ }^{4}$ and the capability of controlling their size, shape, and composition by colloid chemistry brings new opportunities to develop novel nanocatalysts. ${ }^{5,6}$

Colloidal nanoparticles synthesis involves the reduction of metal ions in solution, and since the surface energy of finely divided matter is high, freshly precipitated metallic particles naturally tend to aggregate. In order to overcome that problem, freshly prepared nanoparticles are usually precipitated in a solution that contains an organic compound, which will adhere to their surface and keep the particles suspended. ${ }^{7}$ After transfer to a solid support, this capping layer remains at the surface of the particles. It is known, however, that at the molecular scale, the organic capping layer contains open spaces that permit the reactant and product molecules to adsorb and desorb from the surface of the catalytically active metal. Earlier studies indicate that catalytic activity highly depends on the nature of the interaction between the molecules and the metal sites ${ }^{8,9}$. However, the 
role of the capping agent in the catalytic activity and selectivity is poorly understood in spite of its importance in heterogeneous catalysis as an emerging model system.

Sum frequency generation vibrational spectroscopy (SFGVS) is an important tool in the basic understanding of catalysis at the molecular level since it is able to identify reaction intermediates under catalytic conditions. Several SFGVS studies on catalytic reactions, including $\mathrm{CO}$ oxidation over colloid nanoparticles, were carried out earlier. The vibrational signature of CO does not overlap with the capping agent vibrational modes, facilitating the interpretation of the resulting spectra. ${ }^{10-12}$ Other reactions, such as pyridine hydrogenation, were also investigated, although only outside of the methylmethylene stretch vibrational region. ${ }^{13}$ Reactions more relevant to the industrial processes of hydrogenation, cracking, etc, have been performed on platinum single crystals and probed by SFGVS, but have not been carried out on nanoparticles due to the abovementioned difficulty. ${ }^{14,15}$

The approach to investigate the role of capping agents involves the removal of capping layers with UV/ozone $\left(\mathrm{O}_{3}\right)$ treatment. The UV/ozone oxidation has been used for decades as a means of cleaning slightly soiled surfaces for a variety of applications. The method typically uses ultraviolet light that includes the wavelengths of 185 and $257 \mathrm{~nm}$, where the former generates ozone upon interacting with molecular oxygen. The UV/ ozone oxidation process involves the simultaneous action of ozone and ultraviolet light, which are responsible for the oxidation of the carbon containing compounds into carbon dioxide and water. ${ }^{16}$ Ultraviolet radiation can also be used for polymer surface modification with applications in photolithography, microfluidics, and bioengineering, and its surface effects were studied using SFGVS, ${ }^{17,18}$ which was used as well to 
investigate the effect of ultraviolet radiation and ozone on typical organic substances

present in the upper atmosphere. ${ }^{19}$ Earlier results of removal of the capping agent using the same method were furthermore reported for gold nanospheres with satisfactory results, as well as for bimetallic PtFe nanoparticles. ${ }^{20,21}$

In this work, the application of UV/ozone oxidation is used to remove the capping agent so that SFGVS catalysis studies at the surface of nanoparticles catalysts can be carried out. Previous attempts using methods such as plasma cleaning, hydrogen treatment, or heat treatment under different gaseous atmospheres proved inefficient, because of the irreversible modification of the shape and distribution of the nanoparticles. We monitored the removal of the capping agent from the surface of platinum nanocubes with various analytical methods including SFGVS, XPS, and DRIFTS. The shape and spatial distribution of the nanoparticles are examined with SEM and TEM before and after UV/ozone treatment. In addition ethylene hydrogenation at room temperature and atmospheric pressure is performed at the surface of the catalytic nanocubes and the reaction intermediates are probed with SFGVS.

\section{Experimental procedures}

\section{II.a. Synthesis of TTAB or PVP capped nanoparticles.}

The nanoparticles we used in this study consist of $10 \mathrm{~nm}$ platinum cubes capped with tetradecyl trimethylammonium bromide (TTAB), or polivynil pyrrolidone (PVP). TTAB coated platinum nanocubes were synthesized according to literature methods. ${ }^{6}$ 
Aqueous solutions of tetradecyltrimethylammonium bromide $(400 \mathrm{mM}, 2.5 \mathrm{~mL})$ and dipotassium tetrachloroplatinate(II) $(10 \mathrm{mM}, 1 \mathrm{~mL})$ were added to water $(5.9 \mathrm{~mL})$ in a reaction vial at room temperature. The solution was mixed with a vortex mixer for $30 \mathrm{sec}$ and let still until needle shape crystals formed. The mixture was then heated at $50^{\circ} \mathrm{C}$ in oil bath under magnetic stirring until the crystals dissolved. An ice cooled aqueous solution of sodium borohydride $(500 \mathrm{mM}, 0.6 \mathrm{~mL})$ was added rapidly to the solution. The hydrogen gas formed was released via a needle spearing the septum rubber capping the reaction vial. The needle was removed after $15 \mathrm{~min}$ of reaction. The reaction mixture was then magnetically stirred for more than 7 hours at $50^{\circ} \mathrm{C}$. The resulting brown solution was centrifuged at 3000rpm for 30 min to remove the larger Pt nanoparticles. The supernatant solution was separated and centrifuged again at $12000 \mathrm{rpm}$ for $10 \mathrm{~min}$. The precipitate was collected and re-dispersed in deionized water.

The synthetic procedure of PVP capped Pt nanoparticles is described elsewhere. ${ }^{22}$ Briefly, $\quad 0.1$ mmol ammonium hexachloroplatinate $\quad$ (IV), 1.5 mmol trimethyl(tetradecyl)ammonium bromide, and 2 mmol poly(vinylpyrrolidone) were added to $20 \mathrm{ml}$ ethylene glycol in a $50 \mathrm{ml}$ three-necked flask at room temperature. The stock solution was heated to $80{ }^{\circ} \mathrm{C}$ in a Glas-Col electromantle $(60 \mathrm{~W}$; $50 \mathrm{ml})$ with a ColeParmer temperature controller (Diqi-sense ${ }^{\circledR}$ ), and was evacuated at this temperature for 20 min to remove water and oxygen under magnetic stirring. The flask was then heated to $180{ }^{\circ} \mathrm{C}$ at $10^{\circ} \mathrm{C} / \mathrm{min}$, and maintained at this temperature for $1 \mathrm{~h}$ under Ar. When the reaction was complete, an excess of acetone was added at room temperature to form a cloudy black suspension. This suspension was separated by centrifugation at $4200 \mathrm{rpm}$ for $10 \mathrm{~min}$, and the black product was collected by discarding the colorless supernatant. 
The precipitated platinum nanocrystals were washed twice by precipitation/dissolution (re-dispersed in $7.5 \mathrm{ml}$ of ethanol with sonication and then precipitated by adding $37.5 \mathrm{ml}$ of hexanes).

\section{II.b. Analytical methods}

The shape and size distribution of the particles, deposited on a standard silica grid, were monitored with a TEM FEI Tecnai 12 operating at $100 \mathrm{kV}$. For spectroscopic purposes and XPS measurements, the nanoparticles were deposited either by drop casting or by using the Langmuir Blodgett (LB) technique. ${ }^{22}$ The nanoparticle surface monolayer was compressed at a rate of $20 \mathrm{~cm}^{2} / \mathrm{min}$, and the deposition of the nanoparticles was carried out by lifting the substrate previously submerged in the water. The change of morphology of two-dimensional array of PVP coated nanoparticles was characterized with SEM before and after the removal of the polymer capping agent. A Zeiss Gemini Ultra-55 analytical scanning electron microscope was used for this study.

The irradiation of the nanoparticles was conducted ex-situ using a Bulbtronics 16 W low pressure mercury lamp emitting at 185 and $257 \mathrm{~nm}$ inside a custom made metallic enclosure. The sample was positioned at a distance of $5 \mathrm{~mm}$ from the ultraviolet lamp's quartz tube surface for a determined amount of time, and then removed from the UV chamber. The films used for SFG measurements were deposited on the surface of silica prisms which were irradiated and subsequently attached to a custom made SFG reaction cell for in situ spectroscopy in near total internal reflection (TIR) geometry, pressed against a ${ }^{\circledR}$ Kalrez O-ring by a Teflon block with two set screws. For catalytic studies, a 
mixture of 35 Torr ethylene (Matheson 99.98\%), 150 Torr hydrogen (ultrahigh purity Matheson) and argon (ultrahigh purity Matheson balanced to 760 Torr), was introduced to the cell, which is connected to a gas manifold through stainless steel tubing. The mixture was circulated using a recirculation pump (Metal Bellows Div.), in order to maintain a homogeneous concentration and temperature of reactants and products in the reactor volume.

The SFG spectrometer consists of a mode-locked Continuum Nd:YAG, emitting at $1064 \mathrm{~nm}$ with a pulse width of $20 \mathrm{ps}$ and $20 \mathrm{~Hz}$ repetition rate. It generates pulsed beams of tunable infrared $\left(2750-3600 \mathrm{~cm}^{-1}\right)$ and $532 \mathrm{~nm}$ light at angles of $60^{\circ}$ and $55^{\circ}$, respectively, with respect to the sample surface normal. The beam energies are $100 \mu \mathrm{J}$ and $80 \mu \mathrm{J}$ for the tunable IR and the $532 \mathrm{~nm}$ beams, respectively, and they are considerably defocused in order to decrease the energy density due to the size and weak bonding of the nanoparticles to the support, since previous studies have demonstrated that higher energies could remove them from the surface. ${ }^{10}$ The infrared, $532 \mathrm{~nm}$ and sum frequency beams are p-polarized. The sum frequency signal is passed through a monochromator and collected by a photomultiplier tube. The resulting signal is sent to a gated integrator, and the sum frequency output is normalized by the intensity of the infrared beam.

SFG is a second order nonlinear spectroscopy, sensitive to molecules in noncentrosymmetric environments. Even though nanoparticle systems are inherently centrosymmetric, the TIR geometry circumvents the problem due to the evanescent nature of the electric fields as the distance away from the prism surface increases. ${ }^{23,24}$ The second order nonlinear polarization $\boldsymbol{P}^{(2)}{ }_{(\omega l+\omega 2)}$ is described by 


$$
\boldsymbol{P}^{(2)}=\chi^{(2)}: \boldsymbol{E}\left(\omega_{v i s}\right) \boldsymbol{E}\left(\omega_{I R}\right)
$$

equation 1

where $\boldsymbol{E}\left(\omega_{i}\right)$ is the electric field of the incoming beam and $\chi^{(2)}$ is the second order nonlinear susceptibility. The susceptibility has a non-resonant $\chi_{N R}^{(2)}$ and a resonant contribution $\Sigma \chi_{R}{ }^{(2)}$. The $\chi_{N R}{ }^{(2)}$ is a background contribution from the interface, and $\Sigma \chi$ ${ }_{R}^{(2)}$ includes contributions from individual resonant modes $\chi_{R}{ }^{(2)}$. The expression for $\chi_{R}{ }^{(2)}$ is:

$$
\chi_{R}^{(2)}=\left(\frac{N\left\langle\beta^{(2)}\right\rangle}{\omega_{I R}-\omega_{q}+i \Gamma_{q}}\right) \quad \text { equation } 2
$$

Where $N$ is the number of modes contributing to the SFG signal, $\Gamma_{q}$ is the damping constant for the $q^{\text {th }}$ vibrational mode with a frequency $\omega_{q}, \omega_{I R}$ is the frequency of the incoming IR beam, and $\beta^{(2)}$ is the molecular hyperpolarizability averaged over all possible molecular orientations, and contains the Raman polarizability and the IR dipole transition moment. The intensity of the SFG signal is proportional to the square of the absolute value of the nonlinear polarization. ${ }^{25-27}$

\section{Results and discussion}

\section{III.a. TEM and SEM characterization of morphological change of Pt nanoparticles after UV-ozone treatment}

Transmission electron microscopy (TEM) was performed on TTAB and PVP (not shown) coated platinum nanocubes deposited on a carbon grid, in order to characterize the surface before and after the treatment in order to observe if any changes in shape or 
aggregation occur. Figures 1a and 1b show TEM images of the freshly prepared TTAB coated Pt cubes and after 2 hours of UV/ozone treatment, respectively. The distance between particles slightly decreases from an average of $2.5 \mathrm{~nm}$ to an average of $0.9 \mathrm{~nm}$, suggesting a substantial reduction of the capping agent amount, while the shape and size of the particles are conserved. This is in contrast with the method of oxygen plasma treatment, traditionally used to oxidize organic molecules adsorbed on surfaces, which in the case of platinum nanoparticles results in the fusion of the particles, thus irreversibly modifying both shape and distribution.

SEM was performed on films of PVP and TTAB (not shown) coated platinum nanocubes deposited on silicon wafers. Figures 2a and 2b show SEM images of twodimensional array of PVP coated nanocubes taken before, and after 270 minutes of irradiation of UV/ozone. The SEM image reveals individual nanoparticles $12 \mathrm{~nm}$ in size and indicates that the nanoparticles do not agglomerate upon UV/ozone treatment.

\section{III.b. X-ray photoelectron spectroscopy (XPS) characterization of PVP and TTAB coated platinum nanocubes before and after UV/ozone treatment}

The XPS experiments were conducted on nanoparticle films deposited on silicon wafers. Spectra were taken before and after UV/ozone treatment. Figures 3a and 3b show the XPS results for TTAB and PVP coated platinum nanocubes respectively. In figure 3a, the ratio of $\mathrm{Pt} 4 \mathrm{f} / \mathrm{C} 1$ s increases 4.4 times after 2 hours of $\mathrm{UV} / \mathrm{O}_{3}$ treatment. It was not possible to detect nitrogen with XPS for these particles because of the small amount of nitrogen in the TTAB molecule. The changes in the platinum peaks also show that the 
nanoparticles partially oxidize after the treatment. Figure 3b shows the XPS results for the PVP coated nanoparticles. The considerably higher amount of nitrogen in the PVP molecule allows for a detailed analysis of the evolution of the nitrogen 1s peak. The ratio Pt4f/N1s changes from 0.97 for the fresh sample, to 0.055 after 270 minutes of irradiation, suggesting an effective removal of the capping agent. As in the previous case, an oxidation of the platinum metal takes place.

\section{III.c. Diffuse Reflectance Infrared Fourier Transform Spectroscopy (DRIFTS) before and after UV/ozone treatment}

The samples were prepared by repeatedly drop-casting a suspension of nanoparticles onto aluminum foil, and characterized with Diffuse Reflectance Infrared Fourier Transform Spectroscopy (DRIFTS). Figure 4a shows infrared spectra of TTAB coated platinum nanocubes as a function of irradiation time, revealing the rapid disappearance of the 14 carbon alkyl chain, while the quaternary ammonium moieties remain unchanged after 120 minutes of treatment. The spectra exhibit peaks at $3024 \mathrm{~cm}^{-}$ ${ }^{1}\left(\mathrm{~N}^{+}-\mathrm{CH}_{3}\right.$;C-H asym str), $2951 \mathrm{~cm}^{-1}\left(\mathrm{~N}^{+}-\mathrm{CH}_{3}\right.$;C-H sym str), $960 \mathrm{~cm}^{-1}\left(\mathrm{~N}^{+}-\mathrm{CH}_{3} \mathrm{C}-\mathrm{N}\right.$ str $)$,

$2913 \mathrm{~cm}^{-1}$ ( $\mathrm{CH}_{2}$; C-H asym. str.), $2863 \mathrm{~cm}^{-1}$ (CH 3 ; C-H sym str.), $2846 \mathrm{~cm}^{-1}\left(\mathrm{CH}_{2}\right.$; C-H sym str.), $1453 \mathrm{~cm}^{-1}$ ( $\delta \mathrm{CH}_{2}$; def scissor), $1374 \mathrm{~cm}^{-1}$ ( $\delta \mathrm{CH}_{3}$ sym. def.; bend umbrella), $906 \mathrm{~cm}^{-1}\left(\sigma \mathrm{N}^{+}-\mathrm{CH}_{3}\right.$; C-H rocking), and $743 \mathrm{~cm}^{-1}\left(\mathrm{C}_{4} \mathrm{~N}^{+}\right.$; sym. $\mathrm{C}_{4} \mathrm{~N}^{+}$skeletal mode), 507 $\mathrm{cm}^{-1}\left(\delta \mathrm{C}_{4} \mathrm{~N}^{+} ; \mathrm{C}_{4} \mathrm{~N}^{+}\right.$def.).

Figure 4b shows infrared spectra of PVP coated platinum nanocubes as a function of irradiation time. In this case, the vibrational signature in the $\mathrm{C}-\mathrm{H}$ stretch region 
disappears completely after 59 hours of treatment, and the peaks that correspond to the pyrrolidone monomers, ${ }^{28}$ take longer time, but their disappearance is not negligible. The spectra exhibit peaks at $1676 \mathrm{~cm}^{-1}$ (C=O, C-N str.), $1423 \mathrm{~cm}^{-1}$ (C-H deformation of cyclic C-H group), $1280 \mathrm{~cm}^{-1}$ (C-N str.), 3000-2850 cm (C-H str.), and $2063-2068 \mathrm{~cm}^{-1}$ (CO str. region). However, no direct comparison of the degradation times of TTAB and PVP can be established here since there was no quantification of the amount of coated nanoparticles deposited during the drop casting procedure, and the amount of non- bound capping agent, which is usually present in addition to the bound one, even after a thorough washing procedure following the synthesis step. Also, the samples used in the DRIFTS experiments consist of thick, multilayer drop cast films of nanoparticles due to the lower sensitivity of the technique.

\section{III.d. Sum Frequency Generation Vibrational Spectroscopy of Pt nanoparticles after before and after UV/ozone treatment}

Figure 5 shows SFG spectra of TTAB and PVP coated platinum nanoparticle arrays prepared with the Langmuir Blodgett technique, and by dropcasting respectively, at room temperature and under vacuum $\left(\sim 10^{-5}\right.$ Torr $)$, at different irradiation times, and at ppp polarization. The vibrational modes of TTAB (fig. 5a) correspond to the $\mathrm{CH}_{2}$ symmetric stretch $\left(2850 \mathrm{~cm}^{-1}\right), \mathrm{CH}_{3}$ symmetric stretch $\left(2875 \mathrm{~cm}^{-1}\right), \mathrm{CH}_{2}$ asymmetric stretch $\left(2909 \mathrm{~cm}^{-1}\right)$, methyl Fermi resonance $\left(2937 \mathrm{~cm}^{-1}\right)$, and $\mathrm{CH}_{3}$ asymmetric stretch $\left(2970 \mathrm{~cm}^{-1}\right)$ assigned from previous studies on TTAB with FTIR. ${ }^{29}$ This suggests that the SFG resonant signal originates mainly from the 14 carbon alkyl chain, since no 
vibrational contributions arising from the methylene groups directly bound to the nitrogen atom are observed. Additionally, the significant presence of methylene contributions is typical of organic adsorbates on rough surfaces evidencing the disorder of the alkyl chains at the surface. ${ }^{30}$

The vibrational spectrum of PVP coated nanoparticles (fig. 5b) shows the asymmetric $\mathrm{CH}_{2}$ stretch from the ring $\left(2960 \mathrm{~cm}^{-1}\right)$, the symmetric $\mathrm{CH}_{2}$ stretch from the ring $\left(2900 \mathrm{~cm}^{-1}\right)$, and the $\mathrm{C}-\mathrm{H}$ stretch $\left(2860 \mathrm{~cm}^{-1}\right)$, in agreement with previous Raman studies on PVP capped platinum nanoparticles. ${ }^{29}$ The $\mathrm{UV} / \mathrm{O}_{3}$ treatment eliminates the SFG signal after 15 minutes for the TTAB coated particles, and 90 minutes for the PVP coated ones.

\section{III.e. Ethylene hydrogenation on UV-ozone treated nanoparticles as probed by SFGVS.}

The aim of removing the capping agent from the surface of the catalytic nanoparticles is to enable the study of catalytic reactions with SFGVS in the region of carbon-hydrogen stretch vibrational modes $\left(2800-3200 \mathrm{~cm}^{-1}\right)$, which would otherwise be masked by the contribution from the capping agent's own vibrations. A reaction that has been well studied on platinum single crystal surfaces is ethylene hydrogenation due to the molecule's structural simplicity and the few intermediates and product. It is well established that ethylene adsorbs as ethylidine, and di- $\sigma$-bonded species, with $\pi$-bonded ethylene and ethyl intermediates, as probed with SFGVS on Pt (100) and $\operatorname{Pt}(111)$, at conditions of room temperature and at 760 Torr. ${ }^{31,32},{ }^{33}$ The same reaction was tested with 
the current system at atmospheric pressure and room temperature and the results are shown in figure 6 for TTAB capped Pt cubes after UV/ozone treatment. The peaks at 2878 and $2910 \mathrm{~cm}^{-1}$ and $3023 \mathrm{~cm}^{-1}$ correspond to ethylidyne, and di- $\sigma$-bonded species.

It is also worthy mentioning that for the two capping agents studied, the UV/ozone treatment does not seem to completely remove all organic adsorbates as evidenced by the DRIFTS measurements. For TTAB, even though the aliphatic chains are eliminated in a relatively short time, some of the vibrational features of the hydrophilic ammonium moieties remain unchanged even after several hours of treatment. Those moieties show carbon-nitrogen stretches characteristic of simple ammonium cations or tri-methyl amine, in agreement with previous results on thermal degradation of TTAB at the surface of platinum nanoparticles, as shown in the work of Borodko et al., ${ }^{34}$ where it is shown that the aliphatic tails are initially degraded, and above $200^{\circ} \mathrm{C}$, the surface remains coated with a layer of ammonium cations, which is thermo stable up to $350{ }^{\circ} \mathrm{C}$

For PVP, the UV/ozone treatment appears to produce pyrrolidone monomers, according to the DRIFTS analysis, and similarly to previous studies on thermal degradation of PVP on platinum nanoparticles. ${ }^{28}$ However, the surface concentration of those monomers steadily decreases with the time of treatment conversely to the case of thermal treatment, where cross-linking of the polymer chains occurs at $200^{\circ} \mathrm{C}$, as evidenced by an increase of the intensity of the overtone of the carbonyl stretch at 3305 $\mathrm{cm}^{-1}$, leading ultimately to the generation of amorphous carbon. ${ }^{29}$ The UV/ozone approach for capping agent removal from metallic nanoparticles appears thus to be a promising alternative technique to the traditional methods. 
The catalytic study conducted in this work shows many similarities with previous SFGVS studies of ethylene hydrogenation on platinum (100) and platinum (111) single crystals. That reaction has been extensively studied, ${ }^{33}$ and it has been shown that the ratio of the di- $\sigma$-bonded to the ethylidyne peaks is much smaller at the platinum (111) surface than at the platinum (100) single crystals, indicating that there is a higher concentration of ethylidyne at the surface of platinum (111). ${ }^{31}$ In the present investigation, the ratio of peak amplitudes of di- $\sigma$-bonded to ethylidyne is roughly the same compared to the platinum (111) case. This is consistent with the surface structure of the platinum nanocubes that possess the preferential face of (100). ${ }^{6,35}$

\section{Conclusion}

UV/ozone treatment was successfully applied to the surface of platinum nanoparticles capped with TTAB and PVP and the absence of capping layers and preservation of the shape and spatial distribution of the nanoparticles were confirmed with SFGVS, DRIFTS, XPS, SEM, and TEM. After removal of the capping layer, reaction intermediates of ethylene hydrogenation were identified under reaction conditions using SFGVS. The results indicate the similarity between the platinum nanoparticles and the platinum single crystal surface. This constitutes a contribution to bridging the materials gap from single crystals to nanoparticle systems, and allows for future SFGVS studies of catalytic reactions on reasonably clean metallic nanoparticles. However, even though UV/ozone cleaning constitutes a useful tool in the spectroscopic 
study of catalytic reactions on metals, from a catalysis stand-point, its effects are still not fully understood, and currently under investigation.

\section{Acknowledgement}

This work was supported by the Director, Office of Science, Office of Basic Energy Sciences, Division of Chemical Sciences, Geological and Biosciences and Division of Materials Sciences and Engineering of the U.S. Department of Energy under contract No.

DE-AC02-05CH11231. H.S.L gratefully acknowledges the financial aid of Korea Research Foundation Grant funded by Korean Government. 


\section{References}

(1) Somorjai, G., A.; Park, J. Y. Catal. Lett. 2007, 115, 87.

(2) Bell, A. T. Science 2003, 299, 1688.

(3) Unterhalt, H.; Rupprechter, G.; Freund, H.-J. J. Phys. Chem. B 2002, 106, 356.

(4) Somorjai, G. A.; Park, J. Y. Physics Today 2007, 60, 48.

(5) Park, J. Y.; Zhang, Y.; Grass, M.; Zhang, T.; Somorjai, G. A. Nano Letters 2008, 8,673

(6) Lee, H.; Habas, S. E.; Kweskin, S. J.; Butcher, D.; Somorjai, G. A.; Yang, P. Angew. Chem. 2006, 45, 7824.

(7) Humphrey, S. M.; Grass, M. E.; Habas, S. E.; Niesz, K.; Somorjai, G. A.; Tilley, T. D. Nano Letters 2007, 7, 785.

(8) Bartholomew, C. H.; Agrawal, P. K.; Katzer, J. R. Advances in Catalysis 1982, 31, 135.

(9) Park, J. Y.; Lee, H.; Renzas, J. R.; Zhang, Y. W.; Somorjai, G. A. Nano Letters 2008, 8, 2388.

(10) Kweskin, S. J.; Rioux, R. M.; Habas, S. E.; Komvopoulos, K.; Yang, P.; Somorjai, G. A. J. Phys. Chem. B 2006, 110, 15920.

(11) Grunes, J.; Zhu, J.; Yang, M.; Somorjai, G. A. Catal. Lett. 2003, 86, 157.

(12) Waldrup, S. B.; Williams, C. T. Catal. Commun. 2007, 8, 1373.

(13) Bratlie, K. M.; Komvopoulos, K.; Somorjai, G. A. J. Phys. Chem. C 2008, $112,11865$.

(14) Zaera, F.; Somorjai, G. A. J. Am. Chem. Soc. 1984, 106, 2288.

(15) Kliewer, C. J.; Bieri, M.; Somorjai, G. A. J.Phys. Chem. C 2008, 112, 11373.

(16) Vig, J. R. J. Vac. Sci. Technol. A 1985, 3, 1027.

(17) Ye, H. K.; Gu, Z. Y.; Gracias, D. H. Langmuir 2006, 22, 1863.

(18) Zhang, D.; Dougal, S. M.; Yeganeh, M. S. Langmuir 2000, 16, 4528.

(19) Stokes, G. Y.; Buchbinder, A. M.; Gibbs-Davis, J. M.; Scheidt, K. A.; Geiger, F. M. Journal of Physical Chemistry A 2008, 112, 11688.

(20) Pang, S.; Kurosawa, Y.; Kondo, T.; Kawaii, T. Chem. Lett. 2005, 34, 544.

(21) Chen, W.; Kim, J.; Sun, S.; Chen, S. Phys. Chem. Chem. Phys. 2006, 8, 2779.

(22) Zhang, Y.; Grass, M. E.; Habas, S. E.; Tao, F.; Zhang, T.; Yang, P.; Somorjai, G. A. J. Phys. Chem. C 2007, 111, 12243.

(23) Yeganeh, M. S.; Dougal, S. M.; Silbernagel, B. G. Langmuir 2006, 22, 637.

(24) Srivastava, A.; Eisenthal, K. Chem. Phys. Lett. 1998, 292, 345.

(25) Shen, Y. R. The Principles of Nonlinear Optics; John Wiley and Sons: New York, Chichester, Brisbane, Toronto, Singapore, 1984.

(26) Buck, M.; Himmelhaus, M. J. Vac. Sci. Technol. A 2001, 19, 2717.

(27) Bloembergen, N. Nonlinear Optics; W. A. Benjamin Inc.: New York, Amsterdam, 1965. 
(28) Borodko, Y.; Humphrey, S. M.; Tilley, T. D.; Frei, H.; Somorjai, G. A. Journal of Physical Chemistry C 2007, 111, 6288.

(29) Borodko, Y.; Habas, S. E.; Koebel, M.; Yang, P.; Frei, H.; Somorjai, G. A. J. Phys. Chem. B 2006, 110, 23052.

(30) Bordenyuk, A., N.; Weeraman, C.; Yatawara, A., K.; Jayathilake, H. D.; Stiopkin, I.; Liu, Y.; Benderskii, A., V. J. Phys. Chem. C 2007, 111, 8925.

(31) Somorjai, G. A.; McCrea, K. R.; Zhu, J. Top. Catal. 2002, 18, 157.

(32) Cremer, P. S.; Su, X.; Shen, Y.-R.; Somorjai, G. A. J. Am. Chem. Soc.

1996, $118,2942$.

(33) Sheppard, N.; DelaCruz, C. Vibrational spectra of hydrocarbons adsorbed on metals .1. Introductory principles, ethylene, and the higher acyclic alkenes. In Advances in Catalysis, Vol 41; Academic Press Inc: San Diego, 1996; Vol. 41; pp 1.

(34) Borodko, Y.; Jone, L.; Lee, H.; Yang, P.; Frei, H.; Somorjai, G. A. 2008, submitted.

(35) Habas, S. E.; Hyunjoo, L.; Radmilovic, V.; Somorjai, G. A. Nature Materials 2007, 6, 692. 
(a)

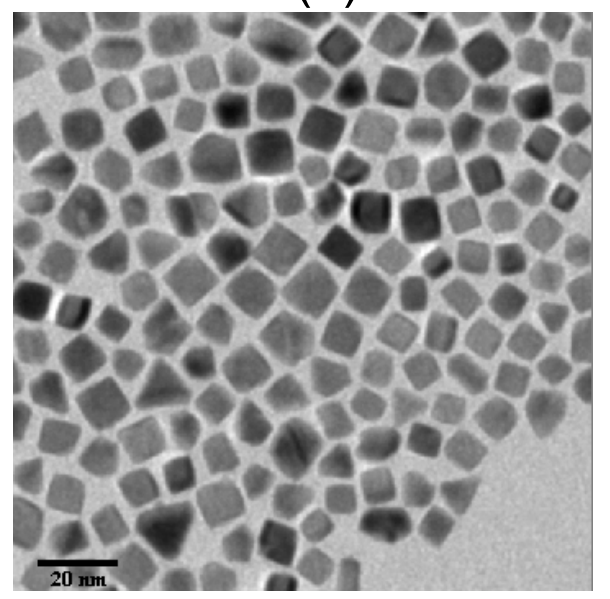

(b)

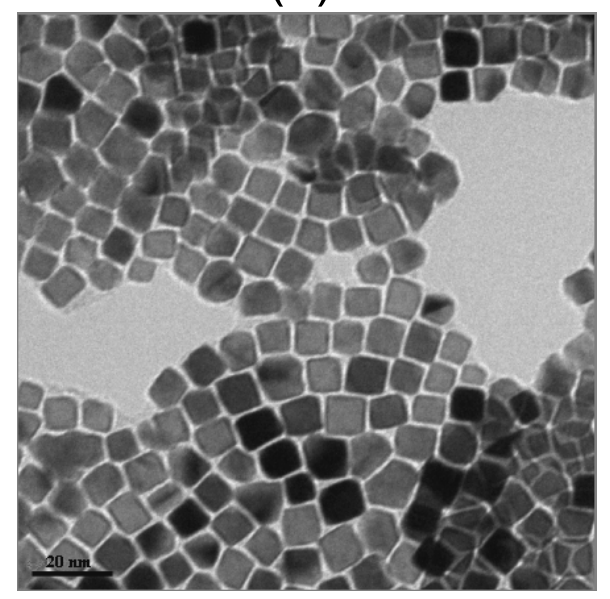

Figure 1. TEM images of a Langmuir-Blodgett film of $10 \mathrm{~nm}$ platinum cubes (a) before and (b) after 2 hours of UV/ozone treatment. 

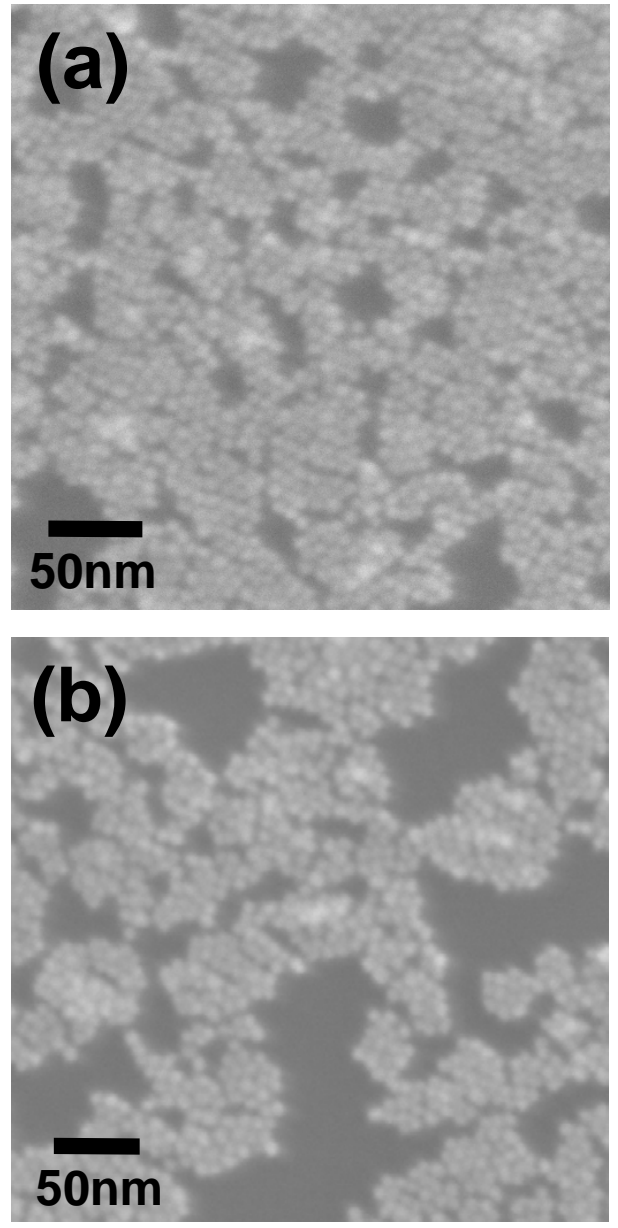

Figure 2. (a) SEM images of PVP coated nanoparticles on silicon wafer (a) before and (b) after UV/ozone treatment for 270 minutes. 

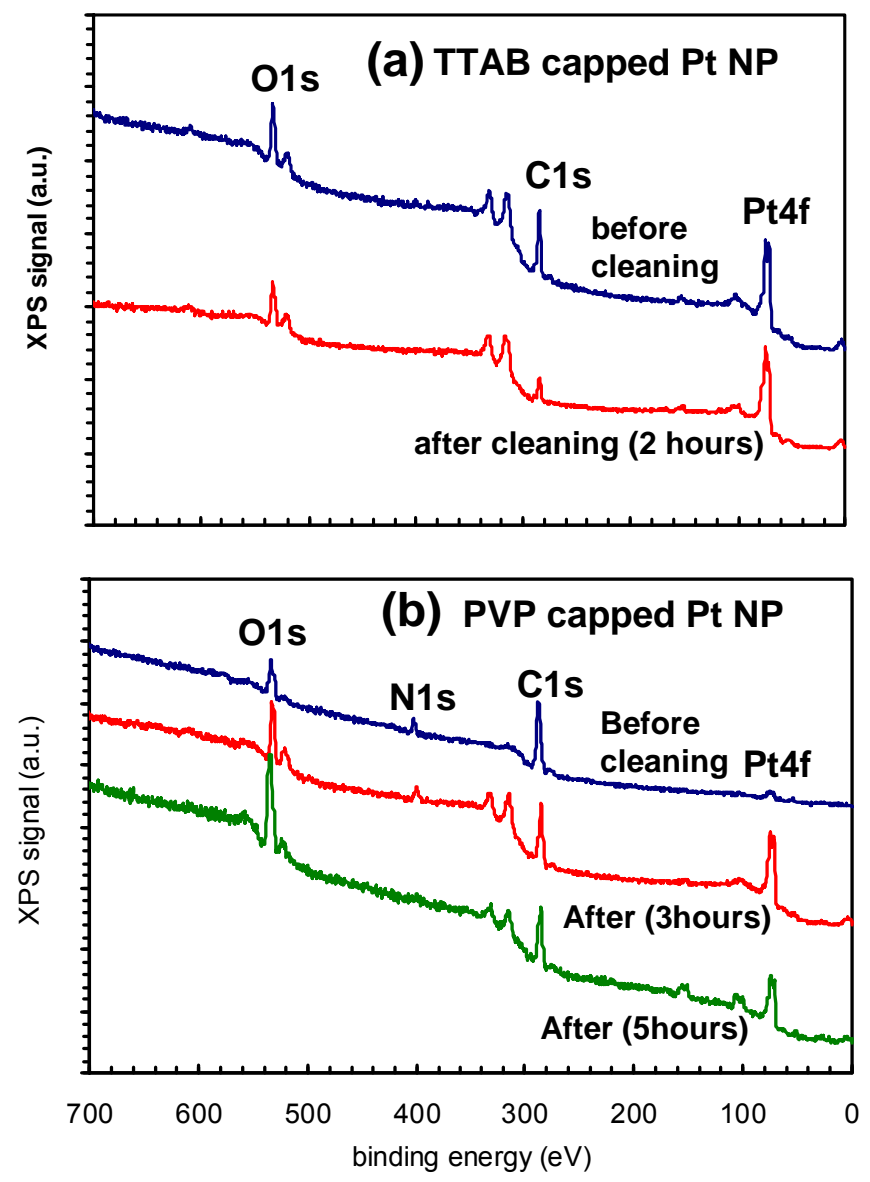

Figure 3. XPS results of (a) TTAB coated, and (b) PVP coated Pt nanocubes deposited on silicon wafer before and after UV-ozone treatment 

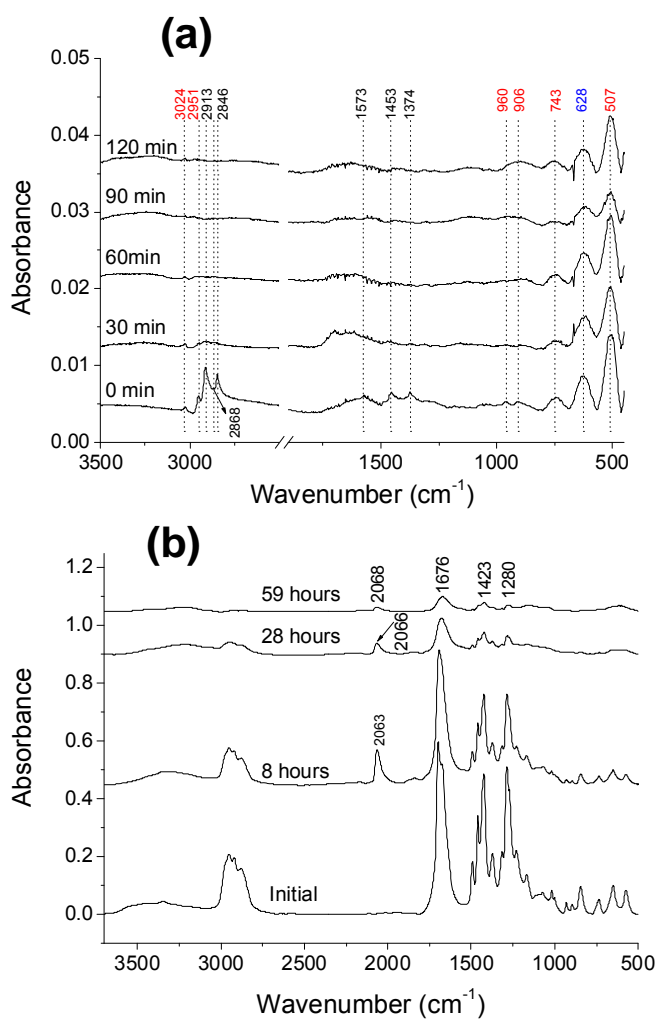

Figure 4. FTIR spectra of (a) TTAB coated platinum nanocubes and (b) PVP coated platinum nanocubes as a function of irradiation time 

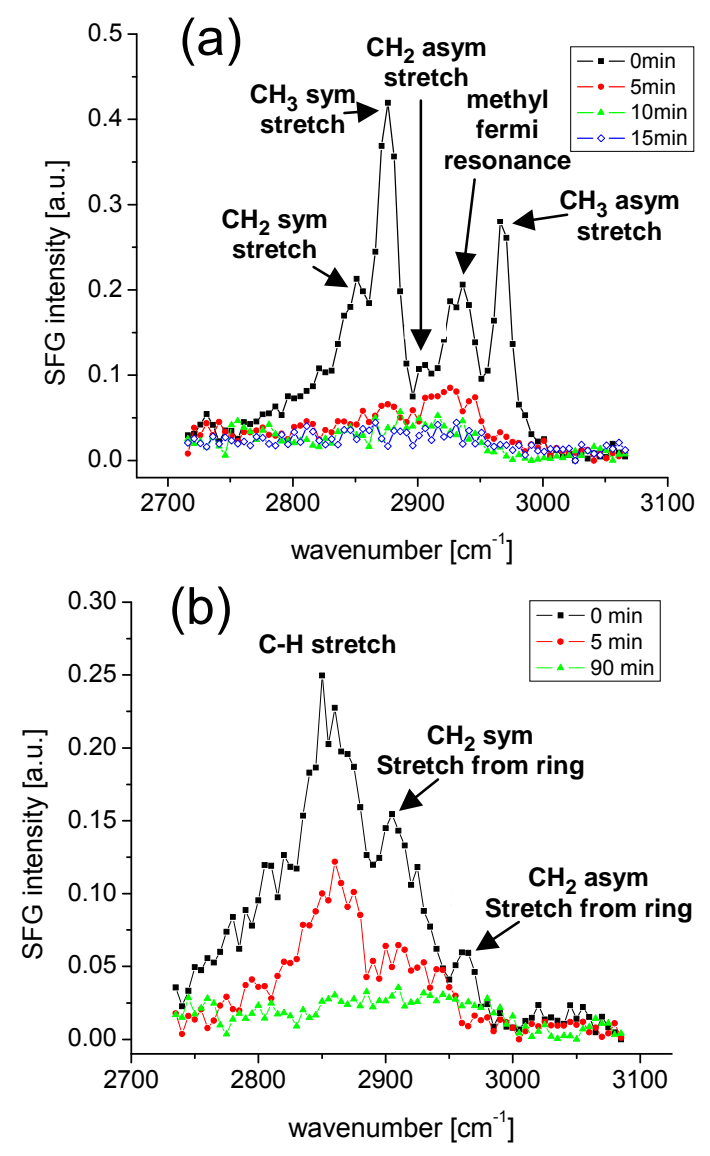

Figure 5. (a) SFGVS spectra of a Langmuir-Blodgett film of $10 \mathrm{~nm}$ TTAB capped platinum cubes. The vibrational modes correspond to the $\mathrm{CH} 2$ symmetric stretch $(2850 \mathrm{~cm}-1)$, CH3 symmetric stretch (2875 $\left.\mathrm{cm}^{-1}\right), \mathrm{CH} 2$ asymmetric stretch (2909 $\left.\mathrm{cm}^{-1}\right)$, methyl fermi resonance (2937 cm-1), and CH3 asymmetric stretch (2970 cm-1). (b) SFGVS spectra of a dropcast film of $10 \mathrm{~nm}$ PVP capped platinum cubes, showing the decrease in signal with irradiation time. The vibrational modes correspond to the asymmetric $\mathrm{CH}_{2}$ stretch from the ring (2960 $\left.\mathrm{cm}^{-1}\right)$, the symmetric $\mathrm{CH} 2$ stretch from the ring $\left(2900 \mathrm{~cm}^{-1}\right)$, and the C-H stretch $(2860 \mathrm{~cm}-1)$. 


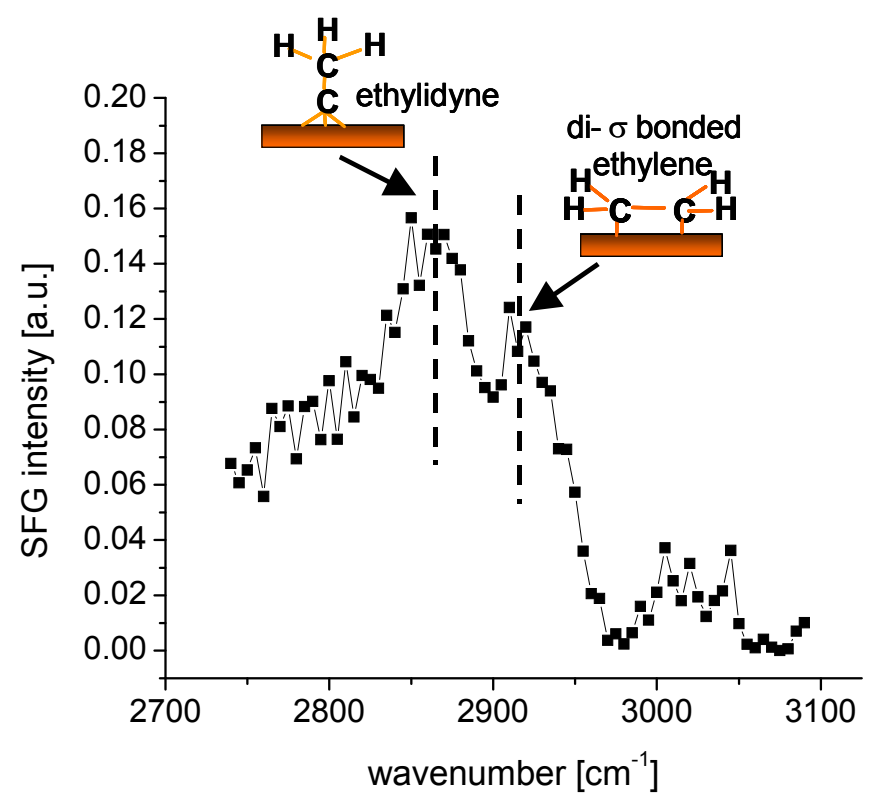

Figure 6. SFG spectra of a drop cast film of $10 \mathrm{~nm}$ PVP capped platinum cube under ethylene hydrogenation condition. The spectrm shows contributions from ethylidine and di- $\sigma$-bonded ethylene adsorbates. A very small contribution from the intermediate $\pi$-bonded species is also visible. At 760 Torr and $298^{\circ} \mathrm{K}$. 